\title{
Some thoughts about food-borne diseases
}

\author{
J. Selva Andina Res. Soc. 2015; 6(1):1.
}

\begin{abstract}
The food-borne diseases - FBD (defined as any syndrome - signs and symptoms - caused by ingestion of food or food ingredients, spices, beverages or water containing etiologic agents in such quantities that affect consumer health, individually or group of people) constitute a serious global problem in public health.

Unhealthy foods ill each year at least two billion people, and can cause death. Although some countries have made great strides in controlling, the disease burden associated with food globally that number is growing. Threats of pollution affecting the food consumed may come from the places where crops are growing or animals are raised and ascend along the chain to the manner in which they are produced, plus the fact many people lack clean water for consumption, which represents a high risk factor for the presentation of gastroenteric diseases.
\end{abstract}

It is very difficult to estimate the true incidence of this disease because sufferers, according to the degree of severity of the clinical picture, not always consult health services and therefore not recorded and reported to the health authorities This situation occurs in both developed countries and even more so in developing countries.

The problem of FBD is not limited to physical damage they cause, although it can sometimes be fatal, but also the negative economic impact that implicitly entails, as it affected both the sick person as the sector of food production and the services of public or private health.

The burden of foodborne diseases is very significant. Better organization and communication between health authorities and consumers would significantly reduce the burden.

The strengthening of national systems of food safety oversight managers along the entire food production chain, operate responsible for producers and processors and active participation of consumers (trained in proper hygienic food preparation) would more effectively manage the supply of safe food for the population.

Claudio Marcelo Zotta Instituto Nacional de Epidemiología "Dr. Juan H. Jara" (INE) Administración Nacional de Laboratorios e Institutos de Salud “Carlos G. Malbrán” (ANLIS) Ministerio de Salud de la Nación Argentina Mar del Plata, Argentina. 0223-4733449. marcelozotta@hotmail.com

2015. Journal of the Selva Andina Research Society. Bolivia. Todos los derechos reservados 